\title{
Mechanisms behind prometastatic changes induced by neoadjuvant chemotherapy in the breast cancer microenvironment
}

This article was published in the following Dove Press journal:

Breast Cancer: Targets and Therapy

\author{
Vladimir M Perelmuter (D) \\ Liubov A Tashireva $\mathbb{D}^{\prime}$ \\ Olga E Savelieva (D) \\ Evgeny $V$ Denisov $\mathbb{1 D}^{1,2}$ \\ Evgeniya $\vee$ Kaigorodova $\mathbb{D}^{1,3}$ \\ Marina $\vee$ Zavyalova (iD) ${ }^{1,4}$ \\ Nadezhda V Cherdyntseva (iD) ${ }^{1,2}$ \\ 'Cancer Research Institute, Tomsk \\ National Research Medical Center, \\ Tomsk 634050, Russia; ${ }^{2}$ Laboratory for \\ Translational Cellular and Molecular \\ Biomedicine, Tomsk State University, \\ Tomsk 634050, Russia; ${ }^{3}$ Department of \\ Biochemistry, Siberian State Medical \\ University, Tomsk 634055, Russia; \\ ${ }^{4}$ Department of Pathological Anatomy, \\ Siberian State Medical University, Tomsk \\ 634055, Russia
}

\begin{abstract}
Chemotherapy, along with surgery and radiotherapy, is a key treatment option for malignant tumors. Neoadjuvant chemotherapy (NACT) reduces the tumor size and enables total tumor resection. In addition, NACT is believed to be more effective in destroying micrometastases than the same chemotherapy performed after surgery. To date, various NACT regimens have been tested and implemented, which provide a favorable outcome in primary tumors and reduce the risk of progression. However, there is increasing evidence of the NACT ability to increase the risk of cancer progression. This review discusses potential mechanisms by which NACT promotes distant metastasis of breast cancer through changes in the microenvironment of tumor cells. We describe prometastatic NACT-mediated changes in angiogenesis, immunoinflammatory reactions in the stroma, intravasation, and amount of circulating tumor cells. The role of NACT-related cellular stress in cancer metastasis is also discussed.
\end{abstract}

Keywords: breast cancer, neoadjuvant chemotherapy, microenvironment, intravasation, metastasis, stress

\section{Introduction}

Neoadjuvant and adjuvant chemotherapy (ACT) is aimed at the destruction of disseminated tumor cells and prevention of recurrence and distant metastasis. Combined regimens that use several chemotherapeutic agents and their combinations with targeted, immune-, and radiotherapy have been developed. This combined treatment leads to improved outcomes in cancer patients. The optimal goal of neoadjuvant chemotherapy (NACT) is to achieve complete regression of the tumor. Numerous studies have demonstrated the reasonability of using NACT for different molecular breast cancer (BC) subtypes. The CTNeoBC analysis, which involved 12 international studies and 11,955 patients, examined the association between pathological complete response (pCR) and disease-free, event-free (EFS), and overall survival (OS). The strongest association between pCR and long-term outcomes was found in patients with aggressive molecular BC subtypes (triple negative; hormonereceptor-positive, high-grade, and HER2-negative; HER2-positive and hormonereceptor-negative). However, an increase in the $\mathrm{pCR}$ rate did not predict an improvement in EFS and OS. The association between pCR and EFS and OS was weakest for low-grade hormone-receptor-positive tumors. ${ }^{1}$

Other meta-analysis compared the effects of NACT and ACT in early stage BC patients. Anthracycline-based chemotherapy was performed in $81 \%$ of the cases. Breast-conserving therapy was used in $65 \%$ of the patients after NACT and $49 \%$ of
Correspondence: Liubov A Tashireva Cancer Research Institute, Tomsk National Research Medical Center, 5 Kooperativny Street, Tomsk 634050, Russia

Tel +7 3822282686 (3147)

Email tashireva@oncology.tomsk.ru 
the patients before ACT. Two-third of NACT patients had a full or partial clinical response. Within 15 years after treatment, local recurrence developed in $21.4 \%$ of the NACT patients and in $15.9 \%$ of the ACT patients. The authors concluded that local recurrences occurred more often after breast-conserving therapy and NACT, despite reduced tumor size. The rate of distant metastasis during this period did not differ between patients with NACT and ACT (38.2\% and $38.0 \%$, respectively). ${ }^{2}$

These results clearly demonstrate that the efficacy of NACT differs significantly among BC subtypes and that even complete tumor regression is not always associated with an increase in EFS and OS. Another conclusion is that NACT is not more effective than ACT in killing of disseminated tumor cells and micrometastases. This explains the lack of differences in the rate of distant metastasis between NACT and ACT.

\section{NACT promotes metastasis via the induction of cellular stress}

Chemotherapeutic drugs are not strictly specific. Their use leads to toxic side effects of varying severity, which precludes further treatment in some cases. A more unfavorable effect of chemotherapy is associated with the appearance of conditions that are critical for cancer progression, not only due to the selection of chemoresistant clones. ${ }^{3}$

Considering chemotherapy as a stressor, chemotherapeutic drugs, eg, paclitaxel, cause changes in both tumor and microenvironment cells. ${ }^{4}$ In response to chemotherapeutic drugs, the activating transcription factor 3 (ATF3) induces expression of numerous transcription factors that are involved in the control of cellular adaptive changes. For example, expression of the cell-cell communication genes and the $M M P 9$ gene is increased in macrophages and results in M2 skew. ${ }^{4,5}$ Increased expression of ATF3 was demonstrated in tumor-stromal cells in BC patients after NACT. ${ }^{4}$ ATF3 activation was found to play a prominent role in mediating the cytotoxicity of doxorubicin in $\mathrm{BC}$ treatment. It is believed that combination of ATF3inducing agents with doxorubicin may be used as a new therapeutic approach. ${ }^{6}$ However, the ATF3-associated effect of chemotherapeutic drugs may lead to opposite results. It was shown that increased expression of ATF3 in non-tumor cells of a mouse BC model induces secondary chemoresistance, an increase in the density of tumor microenvironment of metastasis (TMEM) sites and the number of tumor-infiltrating macrophages, and appearance of metastatic "seeds" (circulating tumor cells, CTCs) and lung metastases. ${ }^{4}$ ATF3 is supposed to be a hub of the cellular adaptive-response network. Many ATF3 target genes encode cytokines and chemokines (eg, CCL2, CCL5, and CXCL2), including those involved in preparing the "soil" and recruiting "seeds" to sites of distant metastases. ${ }^{7}$

A significant role of stress effects in the development of metastases is also confirmed by the fact that non-chemotherapeutic stressors, such as traumatic injury or surgery, also promote metastasis. ${ }^{8}$

\section{Facts on the prometastatic effect of NACT}

The development of distant metastases is associated with the simultaneous occurrence of several conditions. First, the primary tumor should contain "seed" cells and cells capable of producing chemokines that mobilize bone marrow-derived progenitors. Second, the formation of a "soil" (premetastatic niche) is critical for distant metastasis. ${ }^{9}$

In recent years, the possibility of enhanced distant metastasis due to chemotherapy has been discussed. However, in answering the question "Does chemotherapy induce metastasis?", Bruce A. Chabner emphasizes that the supporting results have been obtained primarily in experimental models. ${ }^{10}$ Indeed, there are few clinical studies demonstrating the ability of NACT to increase distant metastasis. Randomized prospective studies have demonstrated that inclusion of paclitaxel in the NACT regimen increases the rate of pCR, but does not improve OS. ${ }^{11}$ In the study by Karagiannis et al, $20 \mathrm{ER}^{+} / \mathrm{HER} 2^{-} \mathrm{BC}$ patients received paclitaxel weekly for 12 weeks, followed by four cycles of doxorubicin and cyclophosphamide. In NACTtreated patients, the primary tumor was evaluated for changes in the TMEM density. TMEM sites were more in the residual tumor than in the tumor biopsy before NACT. ${ }^{12}$ The ability of paclitaxel to enhance metastasis has also been demonstrated in mouse BC models. ${ }^{12,13}$ In in vitro study, treatment of MCF-7 mammary carcinoma cells with adriamycin and/or 5-fluoro-2'-deoxyuridine (FUdR) increased cellular production of interleukin-8 (IL-8) in a dose-dependent manner. IL- 8 is known to promote angiogenesis and epithelial-mesenchymal transition (EMT). ${ }^{14}$ Cells survived after treatment were injected into an orthotopic mouse model. These cells showed faster initial growth and spontaneously metastasize to lungs within 10 weeks compared with the untreated cells. ${ }^{14}$ 


\section{NACT enhances microenvironment- mediated drug resistance}

In some cases, NACT causes the development of secondary chemoresistance that is essential for tumor cells to modulate the tumor microenvironment, exhibit "seed" phenotype, and promote formation of premetastatic niches. Different sensitivity of tumor cells to chemotherapy is one of the manifestations of intratumoral heterogeneity. ${ }^{15}$ For this reason, residual tumor cells can persist locally (in the tumor bed) or as circulating and disseminated tumor cells in other organs. ${ }^{16}$

Chemoresistance of tumor cells is associated with different mechanisms: inhibition of apoptosis, drug efflux from the cell, enhanced DNA repair, EMT, cell dormancy, etc. ${ }^{16}$ In animal models, secondary chemoresistance was shown to be caused not only by genetic alterations in tumor cells but also associated with microenvironmental factors. ${ }^{17}$ For example, gemcitabine resistance develops due to the interaction between tumor cell-derived CXCR4 and the stromal SDF-1/CXCL12. This interaction enables not only adhesion of tumor cells in metastatic niches but also activation of the Akt/PKB and ERK signaling pathways. ${ }^{18}$ Drug resistance may be associated with anti-apoptotic and proliferative microenvironmental signals. Paclitaxel can induce the expression of anti-apoptotic factors (XIAP, IAP-1, IAP-2, Bcl-2, and Bcl-xL) and molecules associated with proliferation (cyclooxygenase 2, c-Myc, and cyclin D1) and metastasis (VEGF, MMP9, and ICAM-1) in tumor cells. ${ }^{19}$ Another mechanism is the integrin a4 $\beta 1$-mediated interaction of tumor cells. This receptor probably plays a key role in de novo resistance. ${ }^{18}$ Integrin $\beta 1$-mediated adhesion of $\mathrm{BC}$ cells to the extracellular matrix reduces paclitaxel-mediated DNA damage and apoptosis and/or induces cell cycle arrest. ${ }^{20}$

Drug resistance of tumor cells may be associated with chemotherapy-induced activation of mesenchymal stem cells (MSCs) in the tumor microenvironment. For example, activation of MSCs during treatment with platinum derivatives was demonstrated in breast, lung, and colon cancer cell lines as well as in xenografts of human breast and esophageal cancer. MSCs secrete polyunsaturated fatty acids that induce resistance of tumor cells to a wide range of chemotherapeutic drugs. Inhibition of the key enzymes (cyclooxygenase- 1 and thromboxane synthase) involved in the production of these acids prevents the development of MSC-induced chemoresistance. ${ }^{21}$

\section{NACT remodels tumor microenvironment}

Distant metastasis is accompanied by complex scenarios of cell-cell and cell-stromal interactions. The tumor microenvironment of malignant tumors, including $\mathrm{BC}$, is represented by a repertoire of different cells: fibroblasts, lymphocytes, neutrophils, macrophages, and other monocyte derivatives, including dendritic cells. The functional state of each of these cells is critical for the origin of metastatic "seeds" and niche-forming cells. Tumor cells participate in remodeling of microenvironment through the production of chemokines and recruiting bone marrow cells that are progenitors of endothelial and hematopoietic cells, fibroblasts, and macrophages. Overall, these cells contribute to angiogenesis and formation of the fibrous stroma.

The microenvironment of solid tumors is characterized by heterogeneity in oxygenation, acidity, proximity of tumor cells to vessels, and the presence of stromal and immune cells. This heterogeneity may underlie differences among tumor cells in proliferation and sensitivity to chemotherapeutics. ${ }^{22}$

Different studies showed that chemotherapy changes the phenotype of stromal and inflammatory cells in the tumor microenvironment. Treatment of co-culture of immortalized human foreskin fibroblasts and MCF7 mammary cancer cells with azathioprine, carboplatin, cisplatin, cyclophosphamide, doxorubicin, 5-fluorouracil, gemcitabine, methotrexate, 6-mercaptopurine, mitoxantrone, 6thioguanine, and taxol led to the activation of fibroblasts and their transformation to the myofibroblast phenotype. However, the effect of these drugs on fibroblasts was different: taxol and doxorubicin induced oxidative stress in fibroblasts, taxol, doxorubicin, and mitoxantrone increased whereas azathioprine reduced IL-6 expression in fibroblasts. Chemotherapy-altered fibroblasts triggered NF-kB and other stress signaling pathways and promoted secretion of IL-6, energy-rich metabolites, and, probably, other ligands that may enhance survival of tumor cells and their potential for metastasis. ${ }^{23}$ In other study, taxotere resulted in overexpression of type IV collagen that protected breast tumor cells from side effects of chemotherapy. Addition of exogenous collagen IV to culture medium attenuated the effect of taxotere on tumor cells. ${ }^{24}$ Wang et al assessed the expression of stromal proteins, THBS1, TNC, FN, SPARC, and $\alpha$-SMA, in BC patients treated by alternation of doxorubicin and docetaxel in NACT. After one or two cycles of 
chemotherapy, expression of these proteins increased in patients with lymph node metastases. An increase in expression of THBS1 and TNC was associated with chemoresistance of tumor cells. ${ }^{25}$

\section{NACT induces tumor angiogenesis}

Tumor angiogenesis is of key importance not only for tumor development but also for distant metastasis. Tumor cells penetrate into blood vessels (intravasation) to colonize other tissues and organs. Microenvironmental signals play a significant role in the regulation of angiogenesis. Chemotherapy was found to induce tumor angiogenesis. In particular, angiogenic effect was shown for paclitaxel. ${ }^{13}$ Cisplatin or paclitaxel enhance expression of VEGFR-1 on endothelial cells in a mouse model of metastasis. Intravenous injection of various tumor cell lines, including $\mathrm{BC}$, causes lung metastases in animal models. According to the authors, this metastasis is related to increased adhesion of tumor cells to the lung vessels that is blocked by anti-VEGFR-1 antibodies. ${ }^{26}$ Plasma collected from paclitaxel-treated mice was able to induce angiogenesis and cause prometastatic effects (eg, cancer cell migration and invasion) in lung and breast carcinoma cells injected into animals. Interestingly, gemcitabine did not have such effect. $^{27}$

Also, chemotherapy can stimulate lymphangiogenesis. For example, paclitaxel was observed to induce VEGF-C expression in tumor-associated macrophages (TAMs) in a mouse model of BC. This activates lymphatic endothelial cells and promotes lymphangiogenesis and metastasis. Blockage of VEGF-C inhibits the activity of chemotherapy-activated macrophages and leads to suppression of lymphangiogenesis. $^{28}$

\section{NACT maintains chronic inflammation in the tumor microenvironment}

Chronic inflammation signs are consistently found in the tumor microenvironment. Cells participating in inflammation are a source of various cytokines, including growth factors, and can affect tumor cells via induction of proliferation, EMT, invasion, and intravasation. In combination, these effects are directly related to metastasis.

Chemotherapeutic drugs, cisplatin, paclitaxel, 5-fluorouracil, and doxorubicin, can cause a proinflammatory effect that promotes metastasis. Paclitaxel is able to activate the TLR-4-MyD88-ERK and NF-kB signaling pathways in
$\mathrm{BC}$ cells that stimulate the production of inflammatory mediators IL-1 $\beta$, IL-8, IL-6, and VEGF-A. IL-6 is an important cytokine that regulates angiogenesis, cell proliferation, and invasion. ${ }^{29}$ Preclinical studies demonstrated the ability of paclitaxel to mediate the development of metastases through TLR4 activation in BC cells. ${ }^{13}$ For example, paclitaxel significantly inhibited the growth of TLR4-negative tumors but promoted metastasis of TLR4-positive tumors to the lymph nodes and lungs. It was found that paclitaxel therapy of patients with TLR4-positive tumors activates systemic inflammatory reactions and contributes to mobilization of myeloid progenitor cells that simultaneously stimulate angiogenesis and lymphangiogenesis both in the tumor and in the premetastatic niches. ${ }^{13}$

In studies on mice bearing 4T1 mammary adenocarcinoma, gemcitabine and 5-fluorouracil activated formation of the NOD-like receptor protein 3 (NLRP3) inflammasome in myeloid-derived suppressor cells (MDSCs). Activation of the inflammasome induced IL- $1 \beta$ production. In turn, IL-1 $\beta$ induced IL-17 secretion by CD4+ T cells. Ultimately, this reduced the efficacy of chemotherapy. ${ }^{30}$ In mouse models with knockout of NLRP3/TNF- $\alpha$ type 1 receptor and IL-1 type 1 receptor, vincristine and doxorubicin synergistically activated NLRP3 inflammasomes and increased expression of IL-1 $\beta$, IL-6, and CXCL1. MAPK inhibitors suppressed the expression of IL-1 $\beta$, IL- 6 , and CXCL1 in the presence of doxorubicin and vincristine alone or together. Thus, specific kinase inhibitors may be useful for reducing inflammation during chemotherapy. ${ }^{30}$

\section{NACT modulates immune responses in the tumor microenvironment}

Immune reactions in the tumor microenvironment are diverse. In spontaneous or induced necrosis, tumor cells release damage-associated molecular patterns that interact with the toll-like receptors of neighboring living tumor cells, normal tissue elements, macrophages, and neutrophils and stimulate the synthesis of proinflammatory cytokines, including chemokines. ${ }^{31}$ As a result, innate-like immune-inflammatory infiltrates appear in the tumor stroma that become the basis for adaptive immune responses to both tumor and non-tumor antigens. ${ }^{32}$ Tumors of the same histological and molecular type differ by a combination of innate and adaptive immune-inflammatory responses. These responses are also heterogeneous within the same tumor. 
It is known that Th1-like immune response occurs with the involvement of the classically activated type 1 macrophages (M1) and shows antitumor activity. In contrast, Th2-like immune response occurs via alternative activated type 2 macrophages (M2) and promotes tumor progression. The dominance of Th1- or Th2-type cytokine effects in certain tumor loci largely determines the risk of distant metastasis and cancer prognosis. ${ }^{33,34}$ The presence of tumor-infiltrating lymphocytes (TILs) in tumors is associated with a better prognosis and a good long-term outcome in patients with various cancers, including BC. ${ }^{35} \mathrm{An}$ increase in the number of TILs and $\mathrm{CD}^{+}$lymphocytes is related to a pCR. ${ }^{36,37}$ Similar data were obtained for TAMs. A greater number of TAMs and TILs in tumor biopsy specimens before NACT are associated with a better outcome after therapy in $\mathrm{BC}$ patients. ${ }^{38}$ However, several studies have reported opposite data regarding the protumor effect of the microenvironment in BC. In particular, carcinoma-associatedfibroblasts (CAFs), MSCs, TAMs, Breg, Treg, and Th2 lymphocytes may contribute to the development of metastases. ${ }^{39-41}$ Litviakov et al reported the lack of clinical response to neoadjuvant anthracycline therapy in $\mathrm{BC}$ patients that was associated with the presence of M2 macrophages (YKL-39- CCL18 ${ }^{+}$ or YKL-39 ${ }^{+} \mathrm{CCL} 18^{-}$) in the tumor. ${ }^{42}$

Several studies showed that NACT can facilitate tumor infiltration by immune cells. NACT with paclitaxel and 5fluorouracil-doxorubicin-cyclophosphamide was found to induce production of CSF-1 and IL-34 by tumor cells. This resulted in the recruitment of CSF1R-positive

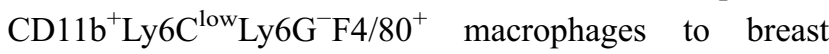
tumors. The inhibition of CSF-1 in human BC led to reduced tumor infiltration by IL-10-positive macrophages and an increase in the response to paclitaxel and carboplatin. Interestingly, this effect depended on the number of $\mathrm{CD}^{+}{ }^{+} \mathrm{T}$ cells. ${ }^{43,44}$ In another study, NACT with CAX (cyclophosphamide, adriamycin, and xeloda), CMX (cyclophosphamide, methotrexate, and xeloda) or taxotere increased infiltration of breast tumors by YKL- $39^{+}$macrophages. This process was associated with an increased risk of distant metastasis. ${ }^{45}$

The available data suggest that chemotherapy can affect differentiation of immune cells. Treatment with trastuzumab in HER2-positive BC was shown to be related to an elevated miR-21 level in dendritic cells that inhibits IL-12 production in these cells and, as a consequence, differentiation of Th1 lymphocytes. ${ }^{46-48}$ In contrast, differentiation of prometastatic Th2 lymphocytes is accelerated through a miR-21-related mechanism. ${ }^{49}$ In addition, miR-21 is able to stimulate the production of IL-6, thereby supporting EMT in tumor cells, as well as affect the tumor immune microenvironment and facilitate cancer progression. ${ }^{50}$ In this regard, the tumor immune microenvironment is a factor that should be considered when prescribing NACT. ${ }^{3}$

\section{NACT affects cancer stemness}

Cancer stem cells are responsible for the maintenance of the bulk-tumor cell proliferation pool, chemoresistance, and tumor dormancy that contributes to delayed metastasis. The plasticity of tumor cells enables to acquire stem properties in certain conditions which can be provided by the immune and stromal microenvironment.

Monocyte chemoattractant proteins (MCPs) are found in the serum of BC patients and associated with chemotherapy-induced monocytosis. In a mouse model, MCPs were shown to induce cancer stem cell formation. ${ }^{51}$ Chemotherapeutics trigger and maintain stem properties in tumor cells by targeting fibroblasts. It was observed that mitoxantrone and taxol activate SMAD and TCF/LEF signaling pathways, whereas carboplatin and doxorubicin enhance STAT3 signaling in tumor cells. ${ }^{23}$ All of these signaling pathways are involved in the control of the stem phenotype in normal and tumor cells. ${ }^{52-54}$ Another study showed that conventional chemotherapy with the maximum tolerated dose (regardless of the drugs used) induces persistent STAT1 and NF- $\mathrm{KB}$ activity in BC-associated fibroblasts. This leads to expression and secretion of $\mathrm{ELR}^{+}$chemokines that bind CXCR2 receptors on cancer cells and trigger the stem properties and invasive behavior. ${ }^{55}$

\section{NACT enhances tumor cell invasiveness}

NACT may force cells of the microenvironment to initiate and accelerate the invasive properties of tumor cells. This can occur through the induction of EMT and production of metalloproteinases and cytokines, including chemokines. For example, MDA-MB-231 BC cells were found to have high adhesive, invasive, and proliferative ability when cocultured with CAFs derived from patients after taxotere chemotherapy. Among 35 genes differentially expressed in CAFs before and after chemotherapy, CXCL2, MMP1, and IL-8 were overexpressed, while RARRES1, FGF1, and $C X C R 7$ were underexpressed. ${ }^{56}$ Different studies showed 
that paclitaxel is capable to accelerate tumor invasiveness. ${ }^{57}$ Paclitaxel increased expression of the actin-regulatory protein MENA ${ }^{\mathrm{INV}}$ in breast tumor xenografts that is attributed to migrating and invasive tumor cells. $^{12}$ In healthy mice, paclitaxel led to the production of IL-1 $\beta$ in macrophages and its accumulation in blood plasma. Treatment of MDA-MB-231 BC cell line with plasma of paclitaxel-treated mice or the IL-1 $\beta$ made tumor cells more invasive. The invasiveness was decreased when tumor cells were treated by plasma of mice received paclitaxel together with the IL-1 $\beta$ inhibitor (Anakinra). Interestingly, the long-term blockade of IL$1 \beta$ or its receptor slightly reduced primary tumor growth, but significantly enhanced spontaneous metastases. It turned out that IL-1 $\beta$ blockage resulted in the differentiation of M1 TAMs into protumor M2 TAMs in the tumor microenvironment, and an increase in vascular permeability, followed by metastasis. ${ }^{58}$ Paclitaxel also demonstrated ability to induce invasion by targeting TIE-2 macrophages. In particular, TIE-2-positive macrophages obtained from mouse breast tumors after paclitaxel stimulated invasion of tumor cells more effectively than TIE-2 macrophages of untreated mice. ${ }^{4}$

\section{NACT promotes intravasation and appearance of CTCs}

Tumor cell intravasation is a key step toward metastasis. Intravasation occurs due to the movement of tumor cells to the vessel in response to chemokine signals generated by perivascular macrophages. ${ }^{4}$ These macrophages together with tumor and endothelial cells are considered as main components of the TMEM. ${ }^{59}$ Robinson et al showed that the TMEM density is greater in patients with distant metastases compared to local BC. In TMEM, macrophages express the angiopoietin receptor TIE-2 and produce VEGF-A and increase vascular permeability that mediates intravasation of tumor cells. In addition, recruited bone marrow-derived circulating cells and TIE-2-expressing monocytes (TEMs) of perivascular regions are involved in paracrine stimulation of angiogenesis. $^{60-64}$ However, TMEM-dependent vascular permeability is not sufficient for intravasation because this process requires the presence of migrating tumor cells. ${ }^{65-67}$ The migrating tumor cells express a large number of prometastatic MENA isoforms, ${ }^{68}$ eg, MENA $^{\mathrm{INV}}$, and a smaller amount of the anti-metastatic MENA11a isoform. ${ }^{66,69}$ Interestingly, Mena knockout mice lack both CTCs and disseminated tumor cells. $^{70}$
Karagiannis et al studied the effect of NACT, in particular paclitaxel or combination of doxorubicin/cyclophosphamide, on the TMEM density in various BC models. ${ }^{12}$ They demonstrated that paclitaxel therapy retarded tumor growth, but simultaneously increased the number of TMEM sites, tumor infiltration by perivascular TIE-2 ${ }^{\text {hi }} /$ VEGF $^{\text {hi }}$ macrophages, TMEM-dependent vascular permeability and mediated tumor dissemination via increased expression of the MENA protein. ${ }^{12}$ Paclitaxel-induced infiltration of macrophages was demonstrated to increase also contact between tumor cells and macrophages. This contact stimulates expression of MENA ${ }^{\mathrm{INV}}$ through activation of the NOTCH signaling pathway and increases TMEM-dependent intravasation. ${ }^{71}$ In another study, Chang et al also reported an increase in the TMEM activity in a mouse BC model after paclitaxel administration. ${ }^{4}$

It is reasonable to suggest that chemotherapy-induced intravasation should increase the number of CTCs. The CTC population is heterogeneous in the ability to initiate metastasis and comprise tumor cells with signs of either EMT or stemness, combination of these signs, and without them. Only $0.01 \%$ of CTCs can initiate metastases, successfully interacting with cellular and molecular components of the premetastatic niche. ${ }^{72}$ The other CTCs either die in the blood or become disseminated tumor cells in the bone marrow and other organs. There is evidence that chemotherapeutic drugs can increase the number of CTCs and therefore promote distant metastasis. For example, in a mouse BC model, paclitaxel was shown to reduce the tumor size, but increase the number of CTCs and pulmonary metastatic burden. ${ }^{4}$ A significant increase in the number of CTCs was observed in patients with HER2-negative BC who received docetaxel/doxorubicin/ cyclophosphamide \pm zoledronic acid in the neoadjuvant regimen and then underwent surgery. However, the number of EpCAM-positive CTCs did not correlate with the pCR in these patients. ${ }^{73}$ Our study showed that NACT influences on the number and population structures of CTCs in BC patients. An increase in CTCs with stem and EMT signs and without membrane EpCAM expression was observed after three NACT courses. ${ }^{74}$

\section{NACT mobilizes bone marrow- derived progenitors and promotes premetastatic niche formation}

Bone marrow progenitor cells are mobilized in response to chemokine signals produced by tumor cells and form 
premetastatic niches in distant tissues and organs. ${ }^{9}$ Besides the mechanisms mentioned above, the prometastatic effect of NACT may be in the release of bone marrow-derived progenitor cells which significantly reduce chemotherapy efficacy. Endothelial progenitor cells (EPCs) were suggested to leave the bone marrow after chemotherapy and enter a tumor where they contribute to angiogenesis. ${ }^{75}$ Indeed, paclitaxel induces proangiogenic mobilization of EPCs in BC patients, whereas gemcitabine lacks this ability. ${ }^{76}$ The number of EPCs in peripheral blood was found to increase in breast, colon, ovarian, esophageal, prostate, head and neck, and cervix cancers after different chemotherapy regimens including platinum drugs, taxanes, anthracyclines, and 5-fluorouracil. An increase in EPCs occurred after 7 and 21 days regardless of the tumor location and the chemotherapy regimen. High number of EPCs after chemotherapy was associated with tumor growth and metastasis and decreased the efficacy of chemotherapy through prevention of necrosis. ${ }^{77}$

The effect of chemotherapy on EPC-mediated angiogenesis was also studied in healthy mice treated with various chemotherapeutics. Paclitaxel, 5-fluorouracil, and docetaxel were found to cause a sharp increase in EPCs within $24 \mathrm{hrs}$ after single bolus injection, while other drugs, such as gemcitabine, cisplatin, and doxorubicin, did not have a similar effect. EPC mobilization was partially mediated by systemic induction of SDF-1a, which can be prevented by blocking anti-VEGFR2 antibodies. ${ }^{76,78}$ Taxane-based chemotherapy was also found to promote tumor growth by stimulating angiogenesis. In particular, taxanes mobilize mesenchymal and endothelial progenitors from the bone marrow. ${ }^{12}$ Paclitaxel enhances the migratory abilities of bone marrow progenitor cells and increases the activity of SDF-1, which leads to recruitment of EPCs, TEMs, and TAMs to the tumor and mobilization of Notchexpressing cells from the bone marrow. ${ }^{57}$ Cisplatin also mobilizes Notch-expressing cells from the bone marrow and leads to increased activity of VEGFR1 in lung endothelial cells. ${ }^{57}$

Chemotherapy was also found to induce the recruitment of MDSCs. In the study by Diaz-Montero et al, different populations of MDSCs (Lin ( $/$ Lo), HLA DR ${ }^{-}$, and $\left.\mathrm{CD} 33^{+} \mathrm{CD} 11 \mathrm{~b}^{+}\right)$were determined before systemic treatment of $\mathrm{BC}$ patients who received doxorubicin-cyclophosphamide every 14 days. Circulating MDSCs were significantly enlarged in patients regardless of the cancer stage compared to healthy individuals. There was also a significant correlation between circulating MDSCs and the clinical cancer stage. In addition, patients with extensive metastatic tumors had the highest percentage and absolute number of MDSCs. ${ }^{79}$

In a mouse $\mathrm{BC}$ model, chemotherapy was shown to contribute to the metastatic spread, providing a fertile ground for metastases. ${ }^{4}$ Paclitaxel and doxorubicin promoted tumor cell secretion of extracellular vesicles (including exosomes) with an increased prometastatic capacity. These vesicles contain annexin A6 (ANXA6) that stimulates NF- $\kappa B$-dependent activation of endothelial cells, induction of CCL2 synthesis, and expansion of Ly $6 \mathrm{C}^{+} \mathrm{CCR} 2^{+}$monocytes in the pulmonary premetastatic niche, thereby promoting lung metastasis. In addition, ANXA6 was found in circulating extracellular vesicles in BC patients undergoing NACT. ${ }^{80}$

\section{Conclusion}

Tumor-stromal interactions are potential targets for chemotherapeutic drugs that either cause hypoxia and death, or alter the functional state of tumor cells and stromal cells, or act as stressors. However, the proportion of these effects in the overall effect of a chemotherapeutic drug is different. As a result, chemotherapeutics cause new microenvironment states in the tumor, the typology of which has been little studied. In some cases, complete or partial regression of the tumor is achieved, or it remains resistant to therapy. However, regardless of the immediate effect of chemotherapy, distant metastases can appear. Furthermore, NACT is able to stimulate distant metastasis (Figure 1).

The prometastatic effect of NACT results from the ability of some chemotherapeutic drugs to stimulate angiogenesis and activity of cancer-associated fibroblasts enhances inflammatory infiltration, and change immune responses in the tumor microenvironment toward the prometastatic Th2 type. In turn, changes in the microenvironment may induce secondary chemoresistance and the appearance of stem-like and invasive properties in tumor cells. Moreover, it leads to an increase in the amount of TMEM, which promotes intravasation, and of CTCs, and recruitment of bone marrow progenitor cells that are necessary for the formation of premetastatic niches. Overall, these changes may provoke distant metastasis.

The results about the increased risk of metastasis during NACT have been mainly obtained in experimental models, and only a few studies have involved BC patients. Further researches are required to investigate the mechanisms of prometastatic effects of various chemotherapeutic 


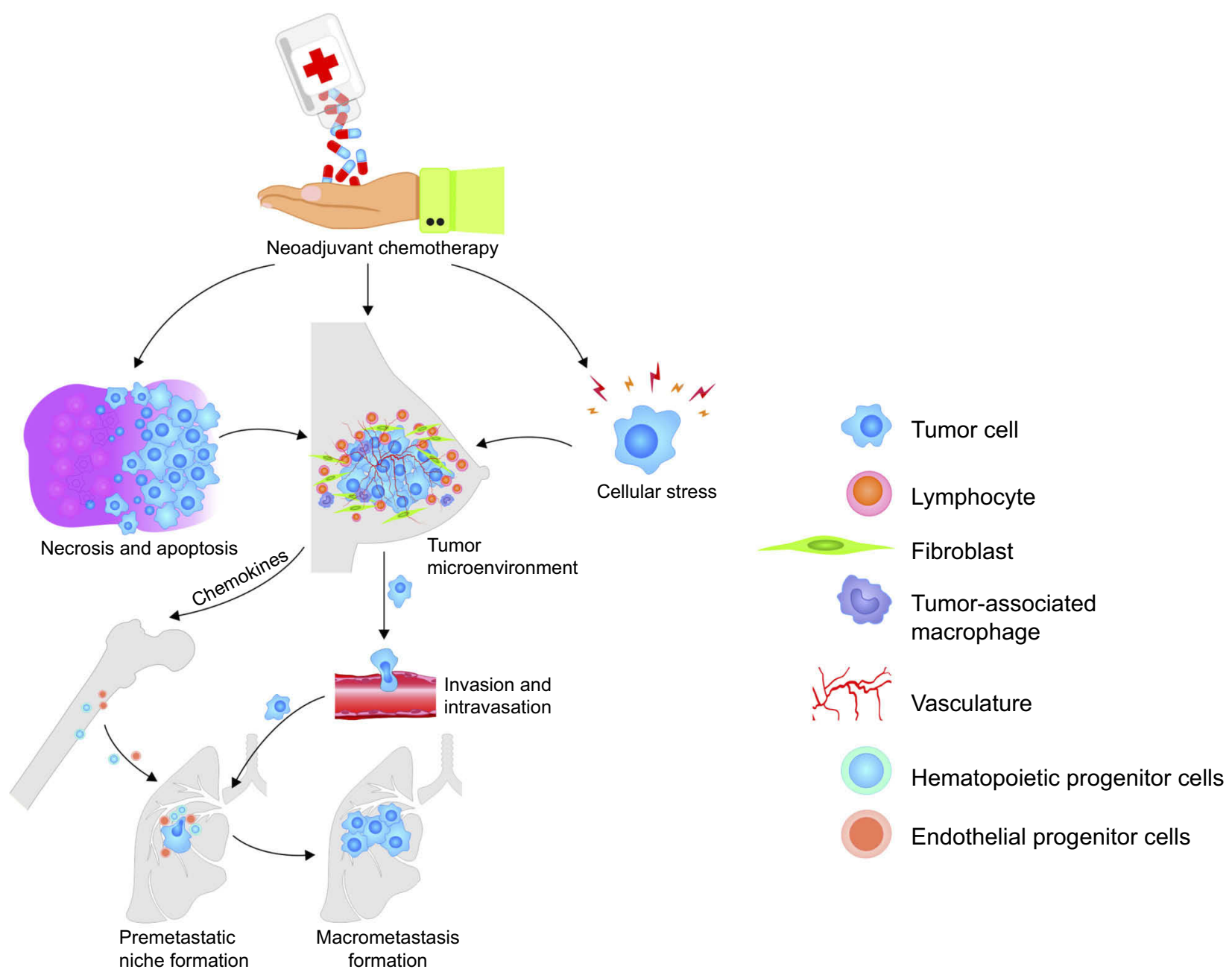

Figure I Mechanisms of prometastatic changes induced by NACT in the BC microenvironment. NACT is able to change the tumor microenvironment through direct and indirect effects. Tumor cell necrosis triggers innate immune-inflammatory reactions that are a background for the development of adaptive immune response. Chemotherapeutics directly impact on the cellular composition of the tumor microenvironment and type of immune-inflammatory reactions whereas their indirect effect is related to the induction of cellular stress. The acceleration of tumor invasiveness and intravasation, as well as an increase in the number of CTCs, are one of the direct and indirect effects of NACT. NACT-stimulated recruitment of bone marrow-derived progenitor cells results in the formation of premetastatic niches and micrometastases. An increase in the number of endothelial progenitor cells and direct stimulation of angiogenesis eventually lead to the development of macrometastases.

Abbreviations: NACT, neoadjuvant chemotherapy; BC, breast cancer; CTCs, circulating tumor cells.

agents used in NACT regimens in $\mathrm{BC}$ and to elucidate the most sensitive stromal elements that are changed in therapy. This will enable to classify chemotherapeutic drugs depending on their ability to alter the microenvironment in $\mathrm{BC}$.

The future belongs to complex neoadjuvant treatment that will include the prescription of drugs based on the molecular BC subtype and individual drug sensitivity as well as accompanying therapy that can modulate or neutralize the prometastatic effects of chemotherapeutics. This therapy should be aimed at the reducing stress, inhibition of the TMEM formation to reduce cancer cell intravasation, correction of the microenvironment to suppress the prometastatic Th2-like response, suppression of recruitment of bone marrow progenitor cells and of their ability to colonize the tumor and premetastatic niches. Accompanying therapy should be personalized based on the initial state of cellular processes that are planning to be targeted. The most important requirement for successful neoadjuvant treatment is the choice of an optimal sequence or synchrony of main chemotherapy and multipurpose accompanying therapy. It does not matter how complicated and costly this complex therapy is, it should be used to eliminate the risk of distant metastasis.

\section{Acknowledgment}

This study was supported by a grant of the Russian Science Foundation (No. 19-75-30016). 


\section{Author contributions}

All authors contributed to data analysis, drafting or revising the article, gave final approval of the version to be published, and agree to be accountable for all aspects of the work.

\section{Disclosure}

The authors report no conflicts of interest in this work.

\section{References}

1. Cortazar P, Zhang I, Untch M, et al. Pathological complete response and long-term clinical benefit in breast cancer: the CTNeoBC pooled analysis. Lancet. 2014;384:164-172. doi:10.1016/S0140-6736(13)62422-8

2. Early Breast Cancer Trialists' Collaborative Group (EBCTCG). Long-term outcomes for neoadjuvant versus adjuvant chemotherapy in early breast cancer: meta-analysis of individual patient data from ten randomised trials. Lancet Oncol. 2018;19:27-39. doi:10.1016/ S1470-2045(18)30144-X

3. Middleton JD, Stover DG, Hai T. Chemotherapy-exacerbated breast cancer metastasis: a paradox explainable by dysregulated adaptiveresponse. Int $J$ Mol Sci. 2018;19(11):3333. doi:10.3390/ ijms19113333

4. Chang YS, Jalgaonkar SP, Middleton JD, Hai T. Stress-inducible gene Atf3 in the noncancer host cells contributes to chemotherapyexacerbated breast cancer metastasis. Proc Natl Acad Sci U S A. 2017;114(34):E7159-E7168. doi:10.1073/pnas.1700455114

5. Wolford CC, McConoughey SJ, Jalgaonkar SP, et al. Transcription factor ATF3 links host adaptive response to breast cancer metastasis. J Clin Invest. 2013;123(7):2893-2906. doi:10.1172/JCI64410

6. Hasim MS, Nessim C, Villeneuve PJ, Vanderhyden BC, Dimitroulakos J. Activating transcription factor 3 as a novel regulator of chemotherapy response in breast cancer. Transl Oncol. 2018;11 (4):988-998. doi:10.1016/j.tranon.2017.10.004

7. Hai T, Wolford CC, Chang YS. ATF3, a hub of the cellular adaptiveresponse network, in the pathogenesis of diseases: is modulation of inflammation a unifying component? Gene Expr. 2010;15:1-11. doi:10.3727/105221610X12819686555015

8. Krall JA, Reinhardt F, Mercury OA, et al. The systemic response to surgery triggers the outgrowth of distant immune-controlled tumors in mouse models of dormancy. Sci Transl Med. 2018;10(436): eaan3464. doi:10.1126/scitranslmed.aao4496

9. Kaplan RN, Riba RD, Zacharoulis S, et al. VEGFR1-positive haematopoietic bone marrow progenitors initiate the pre-metastatic niche. Nature. 2005;438:820-827. doi:10.1038/nature04186

10. Chabner BA. Does chemotherapy induce metastases? Oncologist. 2018;23:273-274. doi:10.1634/theoncologist.2017-0648

11. Gianni L, Baselga J, Eiermann W, et al. Phase III trial evaluating the addition of paclitaxel to doxorubicin followed by cyclophosphamide, methotrexate, and fluorouracil, as adjuvant or primary systemic therapy: European Cooperative Trial in Operable Breast Cancer. J Clin Oncol. 2009;27:2474-2481. doi:10.1200/JCO.2008.19.2567

12. Karagiannis GS, Pastoriza JM, Wang Y, et al. Neoadjuvant chemotherapy induces breast cancer metastasis through a TMEMmediated mechanism. Sci Transl Med. 2017;9(397):eaan0026. doi:10.1126/scitranslmed.aan0026

13. Volk-Draper L, Hall K, Griggs C, et al. Paclitaxel therapy promotes breast cancer metastasis in a TLR4-dependent manner. Cancer Res. 2014;74:5421-5434. doi:10.1158/0008-5472.CAN-14-0067

14. De Larco JE, Wuertz BR, Manivel JC, Furcht LT. Progression and enhancement of metastatic potential after exposure of tumor cells to chemotherapeutic agents. Cancer Res. 2001;61(7):2857-2861.
15. Gerashchenko TS, Denisov EV, Litviakov NV, et al. Intratumor heterogeneity: nature and biological significance. Biochemistry (Mosc). 2013;78(11):1201-1215. doi:10.1134/S00062979 13110011

16. Mordant P, Loriot Y, Lahon B, et al. Minimal residual disease in solid neoplasia: new frontier or red-herring? Cancer Treat Rev. 2012;38:101-110. doi:10.1016/j.ctrv.2011.04.014

17. Blatter S, Rottenberg S. Minimal residual disease in cancer therapy small things make all the difference. Drug Resist Updat. 2015;2122:1-10. doi:10.1016/j.drup.2015.08.003

18. Cukierman E, Bassi DE. The mesenchymal tumor microenvironment: a drug-resistant niche. Cell Adh Migr. 2012;6(3):285-296. doi: $10.4161 / \mathrm{cam} .20210$

19. Aggarwal BB, Shishodia S, Takada Y, et al. Curcumin suppresses the paclitaxel-induced nuclear factor-kappaB pathway in breast cancer cells and inhibits lung metastasis of human breast cancer in nude mice. Clin Cancer Res. 2005;11(20):7490-7498. doi:10.1158/10780432.CCR-05-1192

20. Aoudjit F, Vuori K. Integrin signaling inhibits paclitaxel-induced apoptosis in breast cancer cells. Oncogene. 2001;20:4995-5004. doi:10.1038/sj.onc. 1204554

21. Roodhart JM, Daenen LG, Stigter EC, et al. Mesenchymal stem cells induce resistance to chemotherapy through the release of platinuminduced fatty acids. Cancer Cell. 2011;20(3):370-383. doi:10.1016/j. ccr.2011.08.010

22. Trédan O, Galmarini CM, Patel K, Tannock IF. Drug resistance and the solid tumor microenvironment. $J$ Natl Cancer Inst. 2007;99:1441-1454. doi:10.1093/jnci/djm135

23. Peiris-Pages M, Sotgia F, Lisanti MP. Chemotherapy induces the cancer-associated fibroblast phenotype, activating paracrine Hedgehog-GLI signalling in breast cancer cells. Oncotarget. 2015;6:10728-10745. doi:10.18632/oncotarget.3828

24. Cui Q, Wang B, Li K, et al. Upregulating MMP-1 in carcinomaassociated fibroblasts reduces the efficacy of Taxotere on breast cancer synergized by Collagen IV. Oncol Lett. 2018;16:3537-3544. doi:10.3892/ol.2018.9092

25. Wang T, Srivastava S, Hartman M, et al. High expression of intratumoral stromal proteins is associated with chemotherapy resistance in breast cancer. Oncotarget. 2016;7(34):55155-55168.

26. Daenen LG, Roodhart JM, van Amersfoort M, et al. Chemotherapy enhances metastasis formation via VEGFR-1-expressing endothelial cells. Cancer Res. 2011;71(22):6976-6985. doi:10.1158/0008-5472. CAN-11-0627

27. Gingis-Velitski S, Loven D, Benayoun L, et al. Host response to short-term, single-agent chemotherapy induces matrix metalloproteinase-9 expression and accelerates metastasis in mice. Cancer Res. 2011;71:6986-6996. doi:10.1158/0008-5472.CAN11-0629

28. Alishekevitz D, Gingis-Velitski S, Kaidar-Person O, et al. Macrophage-induced lymphangiogenesis and metastasis following paclitaxel chemotherapy is regulated by VEGFR3. Cell Rep. 2016;17(5):1344-1356. doi:10.1016/j.celrep.2016.09.083

29. Vyas D, Laput G, Vyas AK. Chemotherapy-enhanced inflammation may lead to the failure of therapy and metastasis. Onco Targets Ther. 2014;7:1015-1023. doi:10.2147/OTT.S60114

30. Bruchard M, Mignot G, Derangere V, et al. Chemotherapy-triggered cathepsin $\mathrm{B}$ release in myeloid-derived suppressor cells activates the Nlrp3 inflammasome and promotes tumor growth. Nat Med. 2013;19:57-64. doi:10.1038/nm.2999

31. Bhatelia K, Singh K, Singh R. TLRs: linking inflammation and breast cancer. Cell Signal. 2014;26(11):2350-2357. doi:10.1016/j. cellsig.2014.07.035

32. Law AM, Lim E, Ormandy CJ, Gallego-Ortega D. The innate and adaptive infiltrating immune systems as targets for breast cancer immunotherapy. Endocr Relat Cancer. 2017;24(4):R123-R144. doi:10.1530/ERC-16-0404 
33. Tashireva LA, Perelmuter VM, Manskikh VN, et al. Types of immune-inflammatory responses as a reflection of cell-cell interactions under conditions of tissue regeneration and tumor growth. Biochemistry (Mosc). 2017;82(5):542-555. doi:10.1134/ S0006297917050029

34. Perelmuter VM, Tashireva LA, Manskikh VN, et al. Heterogeneity and plasticity of immune inflammatory responses in the tumor microenvironment: their role in the antitumor effect and tumor aggressiveness. Biol Bull Rev. 2018;8(5):431-449. doi:10.1134/S2079086418050055

35. Fridman WH, Pagès F, Sautès-Fridman C, Galon J. The immune contexture in human tumours: impact on clinical outcome. Nature Rev Cancer. 2012;12(4):298-306. doi:10.1038/nrc3245

36. Yamaguchi R, Tanaka M, Yano A, et al. Tumor-infiltrating lymphocytes are important pathologic predictors for neoadjuvant chemotherapy in patients with breast cancer. Hum Pathol. 2012;43(10):16881694. doi:10.1016/j.humpath.2011.12.013

37. Oldford SA, Robb JD, Codner D, Gadag V, Watson PH, Drover S. Tumor cell expression of HLA-DM associates with a Th1 profile and predicts improved survival in breast carcinoma patients. Int Immunol. 2006;18(11):1591-1602. doi:10.1093/intimm/dx1092

38. Yoon N, Han KM, Cho SY, et al. Tumor-associated macrophages (TAMs) and tumor-infiltrating lymphocytes (TILs) in pretherapeutic breast cancer core biopsies: anti-tumoral effect of immune cells associated with neoadjuvant chemotherapy. Int J Clin Exp Pathol. 2017;10(2):1738-1746.

39. Guo S, Deng CX. Effect of stromal cells in tumor microenvironment on metastasis initiation. Int J Biol Sci. 2018;14(14):2083-2093. doi: $10.7150 /$ ijbs. 24626

40. Ishigami E, Sakakibara M, Sakakibara J, et al. Coexistence of regulatory $\mathrm{B}$ cells and regulatory $\mathrm{T}$ cells in tumor-infiltrating lymphocyte aggregates is a prognostic factor in patients with breast cancer. Breast Cancer. 2019;26(2):180-189. doi:10.1007/ s12282-018-0910-4

41. Zhang Q, Qin J, Zhong L, et al. CCL5-mediated Th2 immune polarization promotes metastasis in luminal breast cancer. Cancer Res. 2015;75(20):4312-4321. doi:10.1158/0008-5472.CAN-14-3590

42. Litviakov N, Tsyganov M, Larionova I, et al. Expression of M2 macrophage markers YKL-39 and CCL18 in breast cancer is associated with the effect of neoadjuvant chemotherapy. Cancer Chemother Pharmacol. 2018;82(1):99-109. doi:10.1007/s00280018-3594-8

43. DeNardo DG, Brennan DJ, Rexhepaj E, et al. Leukocyte complexity predicts breast cancer survival and functionally regulates response to chemotherapy. Cancer Discov. 2011;1:54-67. doi:10.1158/21598274.CD-10-0028

44. Ruffell B, Chang-Strachan D, Chan V, et al. Macrophage IL-10 blocks CD8+ $\mathrm{T}$ cell-dependent responses to chemotherapy by suppressing IL-12 expression in intratumoral dendritic cells. Cancer Cell. 2014;26(5):623-637. doi:10.1016/j.ccell.2014.09.006

45. Liu T, Larionova I, Litviakov N, et al. Tumor-associated macrophages in human breast cancer produce new monocyte attracting and pro-angiogenic factor YKL-39 indicative for increased metastasis after neoadjuvant chemotherapy. Oncoimmunology. 2018;7(6): e1436922. doi:10.1080/2162402X.2018.1490854

46. Feng YH, Tsao CJ. Emerging role of microRNA-21 in cancer. Biomed Rep. 2016;5(4):395-402. doi:10.3892/br.2016.747

47. Lu TX, Munitz A, Rothenberg ME. MicroRNA-21 is up-regulated in allergic airway inflammation and regulates IL-12p35 expression. $J$ Immunol. 2009;182:4994-5002. doi:10.4049/jimmunol.0802775

48. Lu TX, Hartner J, Lim EJ, et al. MicroRNA-21 limits in vivo immune response-mediated activation of the IL-12/IFN-gamma pathway, Th1 polarization, and the severity of delayed-type hypersensitivity. $J$ Immunol. 2011;187:3362-3373. doi:10.4049/jimmunol.1100967

49. Murugaiyan G, Garo LP, Weiner HL. MicroRNA-21, T helper lineage and autoimmunity. Oncotarget. 2015;6:9644-9645. doi:10.18632/ oncotarget.v6i12
50. De Mattos-Arruda L, Bottai G, Nuciforo PG, et al. MicroRNA-21 links epithelial-to-mesenchymal transition and inflammatory signals to confer resistance to neoadjuvant trastuzumab and chemotherapy in HER2-positive breast cancer patients. Oncotarget. 2015;6 (35):37269-37280. doi:10.18632/oncotarget.5495

51. Liu L, Yang L, Yan W, et al. Chemotherapy induces breast cancer stemness in association with dysregulated monocytosis. Clin Cancer Res. 2018;24:2370-2382. doi:10.1158/1078-0432.CCR-17-2545

52. Sakaki-Yumoto M, Katsuno Y, Derynck R. TGF- $\beta$ family signaling in stem cells. Biochim Biophys Acta. 2013;1830(2):2280-2296. doi:10.1016/j.bbagen.2012.08.008

53. Ayadi M, Bouygues A, Ouaret D, et al. Chronic chemotherapeutic stress promotes evolution of stemness and WNT/beta-catenin signaling in colorectal cancer cells: implications for clinical use of WNTsignaling inhibitors. Oncotarget. 2015;6(21):18518-18533. doi:10.18632/oncotarget. 3934

54. Galoczova M, Coates P, Vojtesek B. STAT3, stem cells, cancer stem cells and p63. Cell Mol Biol Lett. 2018;23:12. doi:10.1186/s11658018-0078-0

55. Chan TS, Hsu CC, Pai VC, et al. Metronomic chemotherapy prevents therapy-induced stromal activation and induction of tumor-initiating cells. J Exp Med. 2016;213:2967-2988. doi:10.1084/jem.20151665

56. Rong G, Kang H, Wang Y, Hai T, Sun H. Candidate markers that associate with chemotherapy resistance in breast cancer through the study on Taxotere-induced damage to tumor microenvironment and gene expression profiling of carcinoma-associated fibroblasts (CAFs). PLoS One. 2013;8:e70960. doi:10.1371/journal.pone.0070960

57. Daenen LGM, Houthuijzen JM, Cirkel GA, Roodhart JML, Shaked Y, Voest EE. Treatment-induced host-mediated mechanisms reducing the efficacy of antitumor therapies. Oncogene. 2014;33:1341-1347. doi:10.1038/onc.2013.94

58. Voloshin T, Alishekevitz D, Kaneti L, et al. Blocking IL1beta pathway following paclitaxel chemotherapy slightly inhibits primary tumor growth but promotes spontaneous metastasis. Mol Cancer Ther. 2015;14:1385-1394. doi:10.1158/1535-7163.MCT-14-0969

59. Robinson BD, Sica GL, Liu YF, et al. Tumor microenvironment of metastasis in human breast carcinoma: a potential prognostic marker linked to hematogenous dissemination. Clin Cancer Res. 2009;15 (7):2433-2441. doi:10.1158/1078-0432.CCR-08-2179

60. Jin DK, Shido K, Kopp HG, et al. Cytokine-mediated deployment of SDF-1 induces revascularization through recruitment of CXCR4+ hemangiocytes. Nat Med. 2006;12:557-567. doi:10.1038/nm1400

61. Kerbel RS. Antiangiogenic therapy: a universal chemosensitization strategy for cancer? Science. 2006;312:1171-1175. doi:10.1126/ science. 1125950

62. De Palma M, Venneri MA, Galli R, et al. Tie2 identifies a hematopoietic lineage of proangiogenic monocytes required for tumor vessel formation and a mesenchymal population of pericyte progenitors. Cancer Cell. 2005;8:211-226. doi:10.1016/j.ccr.2005.08.002

63. Grunewald M, Avraham I, Dor Y, et al. VEGF-induced adult neovascularization: recruitment, retention, and role of accessory cells. Cell. 2006;124:175-189. doi:10.1016/j.cell.2005.10.036

64. Udagawa T, Puder M, Wood M, Schaefer BC, D'Amato RJ. Analysis of tumor-associated stromal cells using SCID GFP transgenic mice: contribution of local and bone marrow-derived host cells. Faseb J. 2006;20:95-102. doi:10.1096/fj.04-3669com

65. Roussos ET, Condeelis JS, Patsialou A. Chemotaxis in cancer. Nat Rev Cancer. 2011;11:573-587. doi:10.1038/nrc3078

66. Dovas A, Patsialou A, Harney AS, Condeelis J, Cox D. Imaging interactions between macrophages and tumour cells that are involved in metastasis in vivo and in vitro. $J$ Microsc. 2013;251:261-269. doi:10.1111/j.1365-2818.2012.03667.x

67. Patsialou A, Bravo-Cordero JJ, Wang Y, et al. Intravital multiphoton imaging reveals multicellular streaming as a crucial component of in vivo cell migration in human breast tumors. Intravital. 2013;2: e25294. doi:10.4161/intv.25294 
68. Goswami S, Philippar U, Sun D, et al. Identification of invasion specific splice variants of the cytoskeletal protein Mena present in mammary tumor cells during invasion in vivo. Clin Exp Metastasis. 2009;26:153-159. doi:10.1007/s10585-008-9225-8

69. Hughes R, Qian BZ, Rowan C, et al. Perivascular M2 macrophages stimulate tumor relapse after chemotherapy. Cancer Res. 2015;75:3479-3491. doi:10.1158/0008-5472.CAN-14-3569

70. Roussos ET, Wang Y, Wyckoff JB, et al. Mena deficiency delays tumor progression and decreases metastasis in polyoma middle-T transgenic mouse mammary tumors. Breast Cancer Res. 2010;12: R101. doi:10.1186/bcr2722

71. Pignatelli J, Bravo-Cordero JJ, Roh-Johnson M, et al. Macrophagedependent tumor cell transendothelial migration is mediated by Notch1/MenaINV-initiated invadopodium formation. Sci Rep. 2016;6:37874. doi:10.1038/srep37874

72. Zhe X, Cher ML, Bonfil RD. Circulating tumor cells: finding the needle in the haystack. Am J Cancer Res. 2011;1(6):740-751.

73. Onstenk W, Kraan J, Mostert B, et al. Improved circulating tumor cell detection by a combined EpCAM and MCAM CellSearch enrichment approach in patients with breast cancer undergoing neoadjuvant chemotherapy. Mol Cancer Ther. 2015;14:821-827. doi:10.1158/15357163.MCT-14-0653

74. Kaigorodova EV, Savelieva OE, Tashireva LA, et al. Heterogeneity of circulating tumor cells in neoadjuvant chemotherapy of breast cancer. Molecules. 2018;23(4):E727. doi:10.3390/molecules23040727
75. Rafii S, Lyden D, Benezra R, Hattori K, Heissig B. Vascular and haematopoietic stem cells: novel targets for anti-angiogenesis therapy? Nat Rev Cancer. 2002;2:826-835. doi:10.103 $8 / \mathrm{nrc} 925$

76. Shaked Y, Henke E, Roodhart JML, et al. Rapid chemotherapyinduced acute endothelial progenitor cell mobilization: implications for antiangiogenic drugs as chemosensitizing agents. Cancer Cell. 2008;14:263-273. doi:10.1016/j.ccr.2008.08.001

77. Roodhart JM, Langenberg MH, Vermaat JS, et al. Late release of circulating endothelial cells and endothelia progenitor cells after chemotherapy predicts response and survival in cancer patients. Neoplasia. 2010;12:87-94. doi:10.1593/neo.91460

78. Liang W-C, Wu X, Peale FV, et al. Cross-species vascular endothelial growth factor (VEGF)-blocking antibodies completely inhibit the growth of human tumor xenografts and measure the contribution of stromal VEGF. J Biol Chem. 2006;281:951-961. doi:10.1074/jbc. M508199200

79. Diaz-Montero CM, Salem ML, Nishimura MI, Garrett-Mayer E, Cole DJ, Montero AJ. Cancer increased circulating myeloidderived suppressor cells correlate with clinical cancer stage, metastatic tumor burden, and doxorubicin-cyclophosphamide chemotherapy. Immunol Immunother. 2009;58(1):49-59. doi:10.1007/ s00262-008-0523-4

80. Keklikoglou I, Cianciaruso C, Güç E, et al. Chemotherapy elicits prometastatic extracellular vesicles in breast cancer models. Nat Cell Biol. 2019;21:190-202. doi:10.1038/s41556-018-0256-3
Breast Cancer: Targets and Therapy

\section{Publish your work in this journal}

Breast Cancer - Targets and Therapy is an international, peer-reviewed open access journal focusing on breast cancer research, identification of therapeutic targets and the optimal use of preventative and integrated treatment interventions to achieve improved outcomes, enhanced survival and quality of life for the cancer patient
The manuscript management system is completely online and includes a very quick and fair peer-review system, which is all easy to use. Visit http://www.dovepress.com/testimonials.php to read real quotes from published authors. 